\title{
Eccentric Planets \& Transit Time Variation
}

\author{
David M. Kipping ${ }^{1,3}$ and Ignasi Ribas ${ }^{2,3}$ and Andreu Font-Ribera ${ }^{2,3}$ \\ ${ }^{1}$ Dept. of Physics \& Astronomy, UCL, Gower Street, London, WC1E 6BT, UK \\ email: d.kipping@ucl.ac.uk \\ ${ }^{2}$ Institut de Ciènes de l'Espai (CSIC-IEEE), Campus UAB, 08193, Bellaterra, Spain \\ ${ }^{3}$ The HOLMES Collaboration
}

\begin{abstract}
For an extrasolar planet on an eccentric orbit, the orbital velocity is constantly changing, even during a planetary transit. This changing orbital velocity will, in general, cause lightcurve assymetry. The asymmetry causes the mid-transit time to be slightly off-centre from the halfway point between transit ingress and egress. For GJ436b, we estimate that the midtransit time is shifted by 20 seconds. In the case of a system experiencing secular changes, this difference will lead to a long period transit time variation (L-TTV) signal, under the typical definition of the mid-transit time. In this work, we describe the origins of the effect and evaluate it in the case of GJ436b experiencing hypothetical secular changes. We predict L-TTV could be used to map secular changes in such systems.
\end{abstract}

\section{Introduction}

As the number of planets discovered to be transiting their host star rises with each year, so too does the realization that many of these planets are on eccentric orbits. Like many aspects of exoplanets, observed patterns differ wildly from that which we observe within our own solar system. The analysis of the lightcurves from transiting eccentric exoplanets requires a detailed geometric model and was recently explored by Kipping (2008) (K08).

Previous models for these lightcurves, such as Tingley \& Sackett (2005) and Ford et al. (2008), worked under the assumption that the exoplanet in question maintained a constant velocity during the transit. Although this greatly simplifies the mathematics, it omits the inherent feature of eccentric planets: $\dot{\omega} \neq 0$. A changing orbital velocity during transit introduces lightcurve asymmetry. With this in mind, one can see that the ingress and egress can have different durations.

\section{Shift in $T_{M I D}$}

The model by K08 included the effect of $\dot{\omega} \neq 0$ and as a result deduced that the moment the planet crosses the star's midpoint, $T_{M I D}$ is not the same moment as the halfway point between planetary ingress and egress. We define:

(a) $T_{M I D, t r u e}$ is the moment when the planet centre crosses the star's central line.

(b) $T_{M I D, \text { apparent }}$ is the moment halfway in-between ingress egress.

In the case of a circular orbit, these two definitions are the same. In K08, it was shown that the two definitions may be written mathematically as:

$$
T_{M I D, t r u e}=t_{R E F}+\frac{\mu a^{2}}{J}\left[D\left(f_{m i d}\right)-D\left(f_{1}\right)\right]
$$



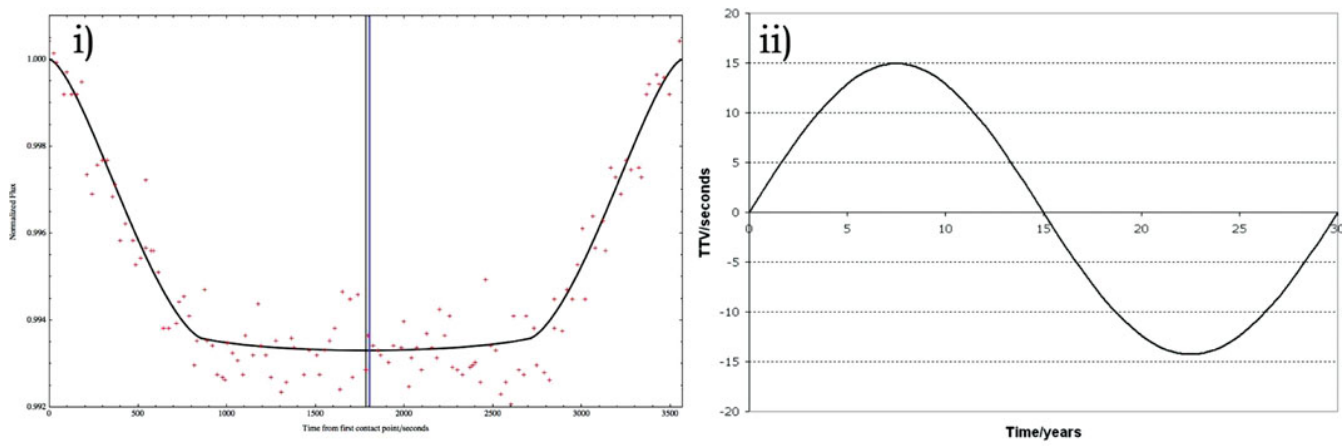

Figure 1. i) GJ436b best-fit lightcurve showing the position of the apparent mid-transit point (left vertical line) and the true mid-transit point (right vertical line); a difference of 20 seconds. ii) Hypothetical L-TTV for GJ436b based on eccentricity changing by 0.1 over a 30 year scale.

$$
T_{M I D, \text { apparent }}=t_{R E F}+\frac{\mu a^{2}}{J} \frac{\left[D\left(f_{4}\right)-D\left(f_{1}\right)\right]}{2}
$$

where $f_{m i d}$ is the true anomaly of the planet when it crosses the star's central line, $f_{1} \&$ $f_{4}$ are the true anomalies at contact points $1 \& 4, \mu$ is the reduced mass, $a$ is the orbital semi-major axis, $J$ is the angular momentum of the planet and $D(f)$ is the duration function, defined in the K08).

We further define: $\Delta T_{M I D}=T_{M I D, t r u e}-T_{M I D, \text { apparent }}$. For GJ436b, we find that $\Delta T_{M I D} \simeq 20$ s. It may be shown that $\Delta T_{M I D}$ is a function of eccentricity, inclination and position of pericentre. If the target system is experiencing secular changes, perhaps due to a perturbing body, then $\Delta T_{M I D}$ will also vary.

Ergo, if one makes continuous measurements of $T_{M I D, \text { apparent }}$ in such systems, they will find that they are not separated by exactly one orbital period, i.e. there is a transit time variation on the time-scale of the secular changes. This measurement can be used to map the secular changes occurring and perhaps infer perturbing bodies. We label this effect long period transit time variation (L-TTV).

We emphasize here a subtle point; L-TTV is a systematic error effect originating from the mid-transit time being typically defined as being halfway in-between ingress and egress. In figure 1, we show i) the best-fit lightcurve for GJ436b (from Spitzer $8 \mu m$, courtesy of Gillon et al. (2007)) with the $T_{M I D, t r u e}$ and $T_{M I D, \text { apparent }}$ lines displayed ii) a hypothetical L-TTV signal for GJ436b where orbital eccentricity is varying sinusoidally over a 30 year time-scale with amplitude 0.1. The hypothetical secular changes in the system were predicted using a 3-body dynamical simulation with the Mercury package. It is interesting to note that even a mildly eccentric planet can produce a detectable effect.

\section{References}

Ford, E. B., Quinn, S. N. \& Veras, D. 2008, ApJ, 678, 1407

Ford, M. et al. 2007, A\&A A, 471, L51

Kipping, D. M. 2008 (K08), MNRAS In press (arXiv:0807.0096)

Tingley, B. \& Sackett, P. D. 2005, ApJ, 631, 1215 